\title{
FACTORES ASOCIADOS A LA DESNUTRICIÓN EN LA GUAJIRA, COLOMBIA
}

\author{
Nelson Jose Alvis-Zakzuk \\ Instituto Nacional de Salud / Universidad de la Costa \\ Colombia
}

Carlos Castañeda Orjuela Instituto Nacional de Salud Colombia

Diana Díaz Jiménez Instituto Nacional de Salud Colombia

Fernando de la Hoz Restrepo Universidad Nacional de Colombia Colombia 


\section{Factores asociados a la desnutrición en La Guajira, Colombia}

\section{Resumen}

La desnutrición infantil produce daños físicos e irreversibles en la capacidad cognitiva, trastornos del crecimiento, retrasos motores y cognitivos, disminución de la inmunidad y un aumento de la morbilidad y mortalidad. Este estudio estimó los factores asociados a la desnutrición en menores de cinco años en La Guajira, Colombia. Se realizó un análisis multivariado de corte transversal, a partir de la Encuesta Nacional de Salud Nutricional (ENSIN). Se estimó un modelo logit sobre los determinantes de la desnutrición global. La variable dependiente se construyó como el peso para la edad menor a dos desviaciones estándar. Se estimaron Odds Ratio (OR) ajustados por pesos muestrales para evaluar el riesgo de las variables independientes con sus valores de referencias. Entre los principales hallazgos, se estudiaron 622 niños menores de cinco años de La Guajira, de los cuales 52,7\% fueron hombres y 55\% vivían en zona rural. El $11,2 \%$ de los niños presenta desnutrición global. Ser el sexto hijo o posterior representa un mayor riesgo de desnutrición global (OR=4,07, IC95\% $=1,50-10,99)$, mientras que habitar viviendas con servicio de alcantarillado los protege de sufrir este tipo de desnutrición $(\mathrm{OR}=0,16$, IC95\%=0,05-0,57). No contar con servicio de alcantarillado y pertenecer a una familia numerosa resultan siendo características de contexto que se asocian con un mayor riesgo de desnutrición infantil en esta región. Afectar estos determinantes sociales permitirá hacer más eficiente la política de vigilancia y control de la desnutrición en este grupo etario.

Palabras clave: Desnutrición, niños, modelos logísticos, oportunidad relativa, factores socioeconómicos, encuestas nutricionales.

\section{Facteurs associés à la malnutrition en Colombie}

\section{Résumé:}

La dénutrition entraîne des dommages physiques et irréversibles de la capacité cognitive, des troubles de la croissance, des retards moteurs et cognitifs et une diminution de l'immunité. De plus, la dénutrition augmente la morbidité et la mortalité chez les enfants de moins de cinq ans. Nous avons estimé les facteurs associés à la dénutrition chez les enfants de moins de cinq ans à La Guajira, en Colombie. Nous avons effectué une analyse multivariée transversale, en utilisant les données de l'Enquête nationale colombienne sur la situation nutritionnelle (ENSIN, en espagnol). Nous avons estimé un modèle logit sur les déterminants de la malnutrition globale. La variable de résultat était la dénutrition, définie comme un rapport poids / âge inférieur ou égal à deux écarts-types. Nous avons estimé les rapports de cotes (OR) ajustés par le plan de sondage afin d'évaluer le risque de chaque groupe par rapport à un groupe de référence. Nous avons étudié 622 enfants de moins de cinq ans, dont 52,7\% d'hommes et $55 \%$ vivant en milieu rural. Sur ce total, $11,2 \%$ des enfants souffraient de dénutrition mondiale. Le fait d'avoir un sixième enfant augmente le risque de dénutrition globale $(O R=4,07, I C 95 \%=1,50-10,99)$. De plus, le fait de vivre dans des maisons avec un service d'assainissement offre une protection contre la dénutrition mondiale $(\mathrm{OR}=0,16$, IC95\% = 0,05-0,57). En bref, les services d'assainissement et les familles nombreuses étaient associés à la dénutrition à La Guajira, en Colombie. Affecter ces déterminants sociaux de la santé améliorerait la politique de surveillance et de contrôle de la dénutrition chez les enfants de moins de cinq ans.

Mots clés: Malnutrition, enfants, modèles logistiques, odds ratio, facteurs socio-économiques, enquêtes nutritionnelles.

\section{Factors contributing to malnutrition in La Guajira, Colombia. Abstract}

Undernutrition produces physical and irreversible damage in cognitive capacity, growth disorders, motor and cognitive delays and decreased immunity. In addition, undernutrition increases morbidity and mortality in children under-five. We estimated the factors associated with undernutrition in children under-five in La Guajira, Colombia. We conducted a cross-sectional multivariate analysis, using data of the Colombian National Survey of the Nutritional Situation (ENSIN, in Spanish). We estimated a logit model on the determinants of global malnutrition. The outcome variable was undernutrition, defined as weightfor-age lesser or equal than two standard deviation. We estimated Odds Ratios (OR) adjusted by the sample design in order to assess the risk of each group compared to a reference group. We studied 622 children under-five years, of which $52.7 \%$ were men and $55 \%$ lived in rural areas. Out of the total, $11.2 \%$ of the children had global undernutrition. Being of the sixth child onwards increases the risk of global undernutrition $(\mathrm{OR}=4.07, \mathrm{CI} 95 \%=1.50-10.99)$. Also, living at homes with sewerage service provides protection against global undernutrition (OR=0.16, CI95\% $=0.05-0.57$ ). In short, sewerage service and large families were associated with undernutrition in La Guajira, Colombia. To affect these social health determinants would improve the policy of surveillance and control of undernutrition in children under-five years.

Keywords: Malnutrition, children, logistic models, odds ratio, socioeconomic factors, nutrition surveys. 


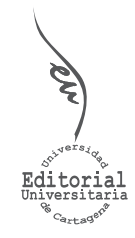

\section{Factores asociados a la desnutrición en La Guajira, Colombia}

INFORMACIÓN DEL ARTíCULO

Recepción del artículo: 20/02/2019

Concepto de evaluación: 30/03/2019

Aceptación del artículo: 15/05/2019
Nelson Jose Alvis-Zakzuk' Instituto Nacional de Salud / Universidad de la Costa, Colombia

Carlos Castañeda Orjuela Instituto Nacional de Salud, Colombia

Diana Díaz Jiménez Instituto Nacional de Salud, Colombia

Fernando de la Hoz Restrepo Universidad Nacional de Colombia, Colombia

\section{INTRODUCCIÓN}

La desnutrición en menores de cinco años es un importante problema de salud pública y tiene serias repercusiones en la morbimortalidad de muchos países del mundo, especialmente aquellos en vía de desarrollo (Black et al., 2013; Sobrino, Gutierrez, Cunha, Davila, \& Alarcon, 2014). La desnutrición infantil produce daños físicos e irreversibles en la capacidad cognitiva, incluyendo trastornos del crecimiento, retrasos motores y cognitivos, disminución de la inmunidad y un aumento de la morbilidad y mortalidad (Kien et al., 2016; Sobrino et al., 2014). Además, puede disminuir la capacidad laboral y la productividad, causando serias repercusiones a nivel económico (Langley-Evans SC, 2015). Según la Organización Mundial de la Salud (OMS), cerca de la tercera parte de las defunciones en niños se asocian con la desnutrición (Organización Mundial de la Salud (OMS), n.d.). En países de ingresos bajos, alrededor del 50\% de las muertes en niños tienen a la desnutrición como su principal causa (De Souza, Petersont, Cufino, do Amaral, \& Gardner, 2001; Luciano Lima Correia et al., 2014).

La mortalidad infantil es un indicador de las condiciones económicas y sociales de un país, así como de la eficiencia de su sistema de salud (Khadka, Lieberman, Giedraitis, Bhatta, \& Pandey, 2015; OECD, 2011). Según el Departamento Administrativo Nacional de 
Estadística (DANE), La Guajira es el departamento con mayor tasa de mortalidad infantil en el Caribe colombiano y uno de los que registra mayor tasa a nivel nacional, siendo el séptimo con mayor mortalidad infantil para 2013 (Departamento Administrativo Nacional de Estadística (DANE), n.d.-b). En Colombia, entre 1998 y 2013 se presentaron 8.928 muertes en niños menores de cinco años a causa de desnutrición, de las cuales el 47,4\% fueron en niñas (Instituto Nacional de Salud - Observatorio Nacional de Salud, 2015). Para 2013, a nivel nacional murieron 6,8 por 100.000 menores de 5 años a causa de desnutrición. La Guajira, con una tasa de mortalidad de 33,4 por 100.000, fue el departamento con mayor mortalidad por desnutrición de la costa caribe colombiana y uno de los de mayor mortalidad por esta causa en Colombia, sólo superado por Chocó, Amazonas, Vaupés, Vichada y Guainía (Instituto Nacional de Salud - Observatorio Nacional de Salud, 2015; Instituto Nacional de Salud, 2016).

Según la Encuesta Nacional de la Situación Nutricional en Colombia (ENSIN) 2010, de acuerdo al bajo peso para la edad, a nivel nacional el 3,4\% de los niños menores de 5 años presentó desnutrición global (Instituto Colombiano de Bienestar Familiar, Instituto Nacional de Salud, Ministerio de Protección Social, \& Asociación Pro-bienestar de la Familia Colombiana, 2005). Esta, fue más prevalente en niños cuyo orden de nacimiento fue 6 o más (8,9\%), en niños con madres sin educación $(12,2 \%)$ y en los indígenas, duplicando la prevalencia nacional para esta última característica (7,5\% vs. 3,4\%) (Instituto Colombiano de Bienestar Familiar et al., 2005).

Para medir la desnutrición se han utilizado tradicionalmente medidas antropométricas que combinan talla, peso y edad como indicadores del estado nutricional basados en estándares de referencia internacional (Johanna, 2010; World Health Organization, 1995). Estas medidas son a su vez explicadas por factores sociodemográficos y económicos que se asocian con su ocurrencia.

En Colombia, la desnutrición puede ser una causa básica o subyacente de mortalidad en una de cada ocho defunciones en menores de un año, y en una de cada tres en infantes entre 1-4 años (Quiroga, 2012). Se han realizado estudios donde se estiman factores asociados a los cambios nutricionales de la población colombiana (Gaviria \& Hoyos, 2011; Karina Acosta Ordoñez, 2012; Moreno, Castro, Paternina, Marrugo, \& Alvis, 2015). Sin embargo, estudios explicativos y multivariados de la desnutrición en el departamento de La Guajira son escasos, aunque importantes, especialmente por ser una región con gran proporción de población indígena que presenta alta vulnerabilidad de su población infantil a los riesgos nutricionales. Teniendo en cuenta lo anterior, el presente estudio estimó los factores asociados a la desnutrición en menores de cinco años en uno de los departamentos con mayor carga de enfermedad debido a desnutrición en Colombia.

\section{MÉTODO}

El presente es un estudio multivariado de corte transversal, desarrollado a partir de los microdatos de la ENSIN 2010 (Instituto Colombiano de Bienestar Familiar et al., 2005). La ENSIN hace un estudio exhaustivo de la situación nutricional en Colombia, evaluando elementos nutricionales y diferentes hábitos de consumo y actividades directamente relacionados con la nutrición (Instituto Colombiano de Bienestar Familiar et al., 2005; Karina Acosta Ordoñez, 2012). Se desarrolló un modelo de regresión binomial para estimar los factores sociodemográficos y económicos asociados a la desnutrición global en niños menores de cinco años de La Guajira. Un departamento que cuenta con una 
población estimada para 2016 de 985.452 personas, de las cuales 129.675 son menores de cinco años, y de estos el 48,9\% son niñas (Departamento Administrativo Nacional de Estadística (DANE), n.d.-a).

\section{Modelación económica de la desnutrición en La Guajira}

Desde el punto de vista microeconómico, existen dos formas de analizar el problema de decisiones individuales nutricionales (Karina Acosta Ordoñez, 2012). En primer lugar, se puede abordar como la producción de un bien (Strauss, 1995) y en segundo, como la demanda de estados de salud (Karina Acosta Ordoñez, 2012; Sahn, Alderman, \& Mason, 1997).

El presente estudio, realizó el análisis desde la óptica de la producción de un bien, el cual resulta de un proceso de maximización de la función de utilidad de los hogares, sujeta a una restricción presupuestaria, donde se incluyen características propias de los niños y variables de su contexto, y se expresa como sigue:

\section{$E N_{i}=\beta_{0}+\beta_{1} X_{i}+\beta_{2} X_{j}+\beta_{3} X_{m}+\mu_{i}$}

En la ecuación (a), el estado nutricional ENi se midió mediante las tallas y los pesos normalizados, además de las probabilidades de sufrir desnutrición.

Xi corresponde a las características relacionadas con el niño, a saber: sexo, edad, orden de nacimiento, etnia (indígena y no indígena), parto prematuro, beneficiario de programa de crecimiento y desarrollo, lactancia y afiliación al sistema general de seguridad social en salud (SGSSS).

$\mathrm{Xj}$ referente a las características relacionadas con el hogar: nivel de escolaridad de la madre, índice de riqueza del hogar y número de personas en el hogar.

Xm agrupa las características relacionadas con la vivienda: acceso a servicio de energía eléctrica, gas natural, acueducto, alcantarillado y área de la vivienda (rural o urbano).

$\mu$ i representa las variables no observadas.

Para estimar los coeficientes de los factores posiblemente asociados a la tasa de desnutrición se estimó un modelo logit sobre los determinantes de la desnutrición global. Las variable dependiente - ENi de la ecuación (a) - incluida el modelo, se construyó de las medidas antropométricas calculadas en la encuesta y sus z-scores. Esta incluye:

Peso para la edad (PPE): se estimó a partir de la normalización del peso del niño y se compara con el correspondiente para su edad y sexo. El déficit de peso para la edad supone la presencia de desnutrición global (Sobrino et al., 2014; UNICEF, n.d.). 
En todos los casos, se utilizó el límite de "menos 2 desviaciones estándar (DE)" como punto de corte: Los niños que se encuentran por debajo presentan déficit nutricional (De Onis M, Garza C, Onyango AW, \& Martorell, 2006; Instituto Colombiano de Bienestar Familiar et al., 2005; Sobrino et al., 2014). Es decir, los niños con PPE <-2 DE presentan desnutrición global, en caso contrario no tienen desnutrición.

En la estimación de los Z-scores para las variables mencionadas inherentes a cada individuo estudiado, se utilizó la metodología de comparación de la característica específica del niño (peso, talla, edad) con las de un niño de referencia, es decir, aquel que tendría las características ideales propuestas por la OMS.

El Z-score se calculó como se indica a continuación:

$$
\text { Zscore }=\frac{\text { Medida individual }- \text { Medida de referencia }(O M S)}{\text { Desviación estandar población de referencia }}
$$

Respecto al análisis de los datos, se utilizaron las bases de datos de antropometría y lactancia de la ENSIN 2010. Los análisis estadísticos se realizaron en el software STATA 12® (Stata Corporation, College Station, TX, USA). El comando "Svy" fue utilizado para ajustar los datos por los grupos de diseño y los pesos de muestreo. Se estimaron frecuencias absolutas y relativas para el análisis descriptivo de las características sociodemográficas y económicas de los niños menores de cinco años pertenecientes al departamento de La Guajira. Para el análisis multivariado, se estimó un modelo de regresión logística (logit), donde la variable dependiente era tener desnutrición global (PPE) frente a no tener desnutrición (dicotómica, valores de 1 y 0 ) y las independientes fueron las variables antes mencionadas. Se calcularon Razones de Odds (OR) para evaluar el mayor o menor riesgo de cada grupo en comparación con el grupo de referencia.

Para efectos de interpretación y análisis de la modelación, la variable índice de riqueza se transformó en dicotómica (quintil 1=1, quintiles 2, 3, 4 y $5=0$ ) y se denominó pobreza. Esta transformación se realizó debido a que algunas categorías no tenían valores. Para la variable orden de nacimiento se usó como punto de comparación ser el primer hijo; en la variable afiliación a salud, se utilizó como referencia estar afiliado a una Empresa Promotora de Salud (EPS) contributiva; en la variable escolaridad de la madre, la referencia fue no tener educación y en la variable personas en el hogar, se utilizó como referencia menos de 6 personas.

\section{RESULTADOS}

En el Cuadro 1 se presentan las características sociodemográficas y económicas de los niños menores de cinco años de La Guajira. En total, se estudiaron 622 niños menores de cinco años, de los cuales 52,7\% fueron hombres y 55\% vivían en zona rural. Aproximadamente, uno de cada tres niños guajiros no estaban afiliados al SGSSS y un 
poco más de la mitad estaban afiliados a Empresas Promotoras de Salud del régimen subsidiado (EPS-S). La mayoría de los niños estudiados habitaban viviendas sin servicio de alcantarillado, gas natural domiciliario y en hogares donde residían 6 personas o más. En cuanto a la escolaridad de la madre, cerca de una de cada cinco no tenían ningún grado de educación. El perfil socioeconómico de los niños evidencia que más de la mitad se encuentran en el primer quintil de riqueza - más pobres.

El 11,2\% de los niños en La Guajira presentó desnutrición global. La prevalencia de desnutrición fue mayor en niños de sexo masculino, de áreas rurales, en los más pobres (primer quintil) y en los no afiliados al SGSSS. De acuerdo con la escolaridad de la madre, entre mayor era el nivel de educación, menor fue la prevalencia de desnutrición global. A su vez, el orden de nacimiento de los niños tuvo una relación positiva con la prevalencia de desnutrición en La Guajira (Figura 1).

Respecto del modelo de la desnutrición global, los resultados del análisis econométrico en niños menores de cinco años de La Guajira se presentan en el Cuadro 2. De acuerdo con las variables explicativas, ser del sexto hijo en adelante representa un riesgo de sufrir desnutrición global (OR=4,07, IC95\%=1,50 - 10,99). Por su parte, habitar viviendas con servicio de alcantarillado, protege de sufrir desnutrición global $(\mathrm{OR}=0,16, \mathrm{IC} 95 \%=$ $0,05-0,57)$.

\section{ANÁLISIS Y DISCUSIÓN}

El presente estudio estimó la asociación entre diferentes variables sociodemográficas y económicas y la ocurrencia de desnutrición global en el departamento de La Guajira, para 2010, a manera de exploración de los determinantes sociales de la salud de este evento. Las características socioeconómicas se han visto extensivamente asociadas con resultados en salud (Campos-Matos, Russo, \& Perelman, 2016; Marmot M, Friel S, Bell R, \& Houweling TA, 2008). Estas variables, son insumos importantes de los estudios de determinantes de la salud, los cuales permiten conocer los principales factores que se asocian al comportamiento de variables sanitarias y definir estrategias para su intervención, desde el punto de vista poblacional.

En nuestro estudio, los hallazgos revelan que el riesgo de sufrir desnutrición global es mayor para aquellas familias numerosas, especialmente del sexto hijo en adelante. A su vez, vivir en viviendas con servicio de alcantarillado disminuye la probabilidad que un niño sufra de desnutrición global. De otra parte, los resultados de los análisis bivariados mostraron que la desnutrición fue mayor en zonas rurales, en el primer quintil de riqueza (más pobres) y en los pertenecientes a etnias indígenas.

Nuestros resultados, para el modelo de desnutrición de niños de La Guajira, son similares a los encontrados en diversos estudios de determinantes de la desnutrición realizados en países de ingresos medios y bajos como Bangladesh, India, Tanzania y Nepal (Fenske, Burns, Hothorn, \& Rehfuess, 2013; Jesmin, Yamamoto, Malik, \& Haque, 2011; Semali, Tengia-Kessy, Mmbaga, \& Leyna, 2015; Tiwari, Ausman, \& Agho, 2014). Por su parte, a nivel teórico, Gary Becker había relacionado también el hecho de tener un orden de nacimiento más elevado con peores niveles y probabilidades de sufrir desnutrición (Becker, 1960; Karina Acosta Ordoñez, 2012). Las relaciones antes mencionadas fueron también importantes hallazgos de nuestra investigación en niños de La Guajira. Por su parte, en un estudio realizado en Perú, se evidenciaron relaciones estadísticamente 
significativas entre la desnutrición y la educación de la madre y el orden de nacimiento (Sobrino et al., 2014), resultados que concuerdan con los del modelo de desnutrición global para el departamento de La Guajira.

La Guajira es el territorio con mayores problemas de desnutrición en la región Caribe colombiana. Esta problemática es reflejo de una variedad de complejas condiciones del contexto que requieren de análisis profundos. El rezago en las condiciones de vida de la población como manifestación de una exclusión histórica que han vivido sus habitantes desde la colonia (Meisel-Roca, 2007); las condiciones climáticas como las bajas precipitaciones que afectan el abastecimiento de agua de consumo y la producción de alimentos (Departamento de la Prosperidad Social - Programa Mundial de Alimentos, n.d.); una estructura económica altamente dependiente de economías extractivas con encadenamientos limitados que tiene un pobre efecto en generación y especialización del empleo (Ministerio de Trabajo, 2015); la precariedad en infraestructura y servicios públicos, incluyendo los de salud (Defensoria del Pueblo, 2014); la comprobada corrupción que acaba con los recursos destinados para la atención de los más vulnerables y comprometen la seguridad alimentaria de la población rural, especialmente de las comunidades indígenas (33); entre otros, son aspectos que contribuyen a la permanencia de esta situación.

Nuestro estudio presenta algunas limitaciones. Al ser un estudio transversal, es difícil definir relaciones de causalidad. Sin embargo, los resultados reflejan algunas asociaciones significativas que permiten crear una idea de los factores que determinan cambios en los riesgos de que un niño esté desnutrido en el departamento de La Guajira. Se resalta también, la pérdida de información (menos del 10\% de los registros) al momento de unir las bases de datos. Lo anterior no afectó la representatividad de la muestra, y por ende de los resultados, debido al tamaño original de la muestra usada por la ENSIN 2010.

Por su parte, referente a la variable explicada en el modelo (PPE), esta se calculó con los z-scores utilizando como comparación la población de referencia de la OMS. El escenario ideal sería construir los z-scores a partir de una población de referencia de niños colombianos, puesto que las características de estos son diferentes a las de una población estándar (Moreno et al., 2015).

\section{CONCLUSIONES}

Las fortalezas del presente estudio se remiten a la utilización de datos provenientes de una encuesta como la ENSIN, que tiene un marco muestral que disminuye los sesgos y genera resultados robustos y representativos de las características de nivel poblacional. Por su parte, los resultados de este estudio resultarán claves al momento de intervenir características sociales y económicas que determinan la desnutrición en La Guajira.

En términos de política pública, conocer los factores asociados con la condición nutricional de los niños en La Guajira es fundamental para optimizar la efectividad de los programas de prevención y control de la desnutrición, a partir de intervenciones que busquen modificar los determinantes sociales de la desnutrición. En tal sentido, los hallazgos del presente estudio destacan la importancia de garantizar los servicios de alcantarillado y energía eléctrica a las viviendas y la necesidad de focalizar esfuerzos para intensificar los programas nutricionales en hogares numerosos. Estas estrategias pueden hacer más eficiente la política de vigilancia y control de la desnutrición. 


\section{Financiación:}

El presente estudio se realizó con recursos de operación del Observatorio Nacional de Salud del Instituto Nacional de Salud.

\section{Conflictos de interés:}

No existen conflictos de interés.

\section{REFERENCIAS BIBLIOGRÁFICAS}

Becker, G. (1960). An Economic Analysis of Fertility. In Demographic and Economic Change in Developed Countries (pp. 225-256). Columbia University Press.

Black, R. E., Victora, C. G., Walker, S. P., Bhutta, Z. A., Christian, P., de Onis, M., ... Child Nutrition Study, G. (2013). Maternal and child undernutrition and overweight in low-income and middle-income countries. Lancet, 382(9890), 427-451. https://doi.org/10.1016/S01406736(13)60937-X

Campos-Matos, I., Russo, G., \& Perelman, J. (2016). Connecting the dots on health inequalities--a systematic review on the social determinants of health in Portugal. Int J Equity Health, 15, 26. https://doi.org/10.1186/s12939-016-0314-z

De Onis M, Garza C, Onyango AW, \& Martorell, R. (2006). Who Child Growth Standards. Acta Paediatr, 95, supl 450:96-101.

De Souza, A. C., Petersont, K. E., Cufino, E., do Amaral, M. I., \& Gardner, J. (2001). Underlying and proximate determinants of diarrhoea-specific infant mortality rates among municipalities in the state of Ceara, north-east Brazil: an ecological study. J Biosoc Sci, 33(2), 227-244.

Defensoria del Pueblo. (2014). Crisis humanitaria en La Guajira.

Departamento Administrativo Nacional de Estadística (DANE). (n.d.-a). Estimaciones de población 1985-2005 y proyecciones de población 2005-2020 nacional y departamental desagregado por área, sexo y grupos quinquenales de edad.

Departamento Administrativo Nacional de Estadística (DANE). (n.d.-b). Estimaciones Tasa de Mortalidad Infantil nacional, departamental y municipal, período 2005 -2013.

Departamento de la Prosperidad Social - Programa Mundial de Alimentos. (n.d.). Conversatorio: "La incidencia de la desertificación, sequía y cambio climático en la seguridad alimentaria y nutricional". Situación de la Alta y Media Guajira, Colombia. 2015.

Fenske, N., Burns, J., Hothorn, T., \& Rehfuess, E. A. (2013). Understanding child stunting in India: a comprehensive analysis of socio-economic, nutritional and environmental determinants using additive quantile regression. PLoS One, 8(11), e78692. https://doi. org/10.1371/journal.pone.0078692

Gaviria, A., \& Hoyos, A. (2011). Anemia and child education: The case of Colombia. Desarrollo y Sociedad, 1(68), 47-77.

Instituto Colombiano de Bienestar Familiar, Instituto Nacional de Salud, Ministerio de Protección Social, \& Asociación Pro-bienestar de la Familia Colombiana. (2005). Encuesta Nacional de la Situación Nutricional en Colombia 2005 - ENSIN.

Instituto Nacional de Salud. (2016). Protocolo de vigilancia en salud pública. Mortalidad por y asociada a desnutrición en $<5$ años. 
Instituto Nacional de Salud - Observatorio Nacional de Salud. (2015). Informe Nacional de las Desigualdades Sociales en Salud en Colombia. Imprenta Nacional de Colombia, Bogotá, D.C, 1, Pag 213.

Jesmin, A., Yamamoto, S. S., Malik, A. A., \& Haque, M. A. (2011). Prevalence and determinants of chronic malnutrition among preschool children: a cross-sectional study in Dhaka City, Bangladesh. J Health Popul Nutr, 29(5), 494-499.

JOHANNA, C. (2010). Factors Contributing to Malnutrition in Children 0-60 Months Admitted to Hospitals in the Northern Cape. University of the Free State Bloemfontein, South Africa.

Karina Acosta Ordoñez. (2012). La desnutrición en los primeros años de vida: Un análisis regional para Colombia. Documentos de trabajo sobre Economía Regional. Banco de la República (CEER) - Cartagena.

Khadka, K. B., Lieberman, L. S., Giedraitis, V., Bhatta, L., \& Pandey, G. (2015). The socio-economic determinants of infant mortality in Nepal: analysis of Nepal Demographic Health Survey, 2011. BMC Pediatr, 15, 152. https://doi.org/https://10.1186/s12887-015-0468-7

Kien, V. D., Lee, H. Y., Nam, Y. S., Oh, J., Giang, K. B., \& Van Minh, H. (2016). Trends in socioeconomic inequalities in child malnutrition in Vietnam: findings from the Multiple Indicator Cluster Surveys, 2000-2011. Glob Health Action, 9, 29263. https://doi.org/10.3402/gha. v9.29263

Langley-Evans SC. (2015). Nutrition in early life and the programming of adult disease: a review. J Hum Nutr Diet, 28(Suppl 1), 1-14.

Luciano Lima Correia, Anamaria Cavalcante e Silva, Jocileide Sales Campos, Francisca Maria de Oliveira Andrade, Márcia Maria Tavares Machado, Ana Cristina Lindsay, ... Cunha, A. J. L. A. da. (2014). Prevalence and determinants of child undernutrition and stunting in semiarid region of Brazil. Rev Saúde Pública, 48(1), 19-28.

Marmot M, Friel S, Bell R, \& Houweling TA. (2008). Closing the gap in a generation: health equity through action on the social determinants of health. Lancet, 372(9650), 1661-9.

Meisel-Roca, A. (2007). La Guajira y el mito de las regalías redentoras. Documentos de Trabajo Sobre Economía Regional y Urbana; No. 86.

Ministerio de Trabajo. (2015). Recomendaciones para promoción del empleo en el marco del trabajo decente a entes territoriales. 2016-2019,1-73.

Moreno, D., Castro, R., Paternina, A., Marrugo, V., \& Alvis, N. (2015). Determinantes sociodemográficos de la nutrición infantil en Colombia. Salud Uninorte, 31(3), 446-457.

OECD. (2011). OECD Factbook 2011-2012: economic, environmental and social statistics. Paris: Organisation for Economic Co-Operation and Development, 1, 268.

Organización Mundial de la Salud (OMS). (n.d.). Informe de estadísticas sanitarias mundiales. 2010.

Quiroga, E. F. (2012). Mortalidad por desnutrición en menores de cinco años, Colombia, 2003-2007. Biomédica, 32(4).

Sahn, D., Alderman, H., \& Mason, P. (1997). On the determinants of nutrition in Mozambique: The importance of age specific effects. World Development, 25(4), 577-588.

Semali, I. A., Tengia-Kessy, A., Mmbaga, E. J., \& Leyna, G. (2015). Prevalence and determinants of stunting in under-five children in central Tanzania: remaining threats to achieving Millennium Development Goal 4. BMC Public Health, 15, 1153. https://doi.org/10.1186/s12889-015-2507-6 
Sobrino, M., Gutierrez, C., Cunha, A. J., Davila, M., \& Alarcon, J. (2014). [Child malnutrition in children under 5 years of age in Peru: trends and determinants]. Rev Panam Salud Publica, 35(2), 104-112.

Strauss, J. (1995). Human resources: Empirical modelling of household and family decisions. Handbook of Development Economics.

Tiwari, R., Ausman, L. M., \& Agho, K. E. (2014). Determinants of stunting and severe stunting among under-fives: evidence from the 2011 Nepal Demographic and Health Survey. BMC Pediatr, 14, 239. https://doi.org/10.1186/1471-2431-14-239

UNICEF. (n.d.). GLOSARIO DE TÉRMINOS SOBRE DESNUTRICIÓN.

World Health Organization. (1995). Physical status: the use and interpretation of anthropometry. Report of a WHO Expert Committee. World Health Organization Technical Report Series. Geneva., 1, 854: 1-452.

\section{Cuadro 1. \\ Características sociodemográficas y económicas de la población en estudio}

\begin{tabular}{|c|c|c|c|c|c|}
\hline Característica & $\mathbf{N}$ & $\%$ & Característica & $\mathbf{N}$ & $\%$ \\
\hline Peso para la edad (PPE) & & & \multicolumn{3}{|l|}{ Afiliación a salud } \\
\hline$<2$ desviaciones estándar & 69 & 11,2 & EPS (contributiva) & 73 & 11,7 \\
\hline \multirow[t]{2}{*}{$>2$ desviaciones estándar } & \multirow[t]{2}{*}{553} & \multirow[t]{2}{*}{88,8} & EPS (subsidiada) & 353 & 56,8 \\
\hline & & & \multirow{3}{*}{$\begin{array}{l}\text { Especial } \\
\text { No está afiliado }\end{array}$} & 7 & 1,1 \\
\hline \multicolumn{3}{|l|}{ Edad } & & 188 & 30,2 \\
\hline 0 & 120 & 19,3 & & & \\
\hline 1 & 122 & 19,6 & \multirow{3}{*}{\multicolumn{2}{|c|}{ Escolaridad de la madre }} & \\
\hline 2 & 127 & 20,4 & & & \\
\hline 3 & 124 & 19,9 & & & \\
\hline \multirow[t]{2}{*}{4} & \multirow[t]{2}{*}{129} & \multirow[t]{2}{*}{20,7} & Sin educación & 106 & 18,0 \\
\hline & & & Primaria & 161 & 27,3 \\
\hline \multicolumn{3}{|l|}{ Sexo } & Secundaria & 237 & 40,2 \\
\hline Hombre & 328 & 52,7 & \multirow[t]{2}{*}{ Superior } & \multirow[t]{2}{*}{85} & \multirow[t]{2}{*}{14,4} \\
\hline Mujer & 294 & 47,3 & & & \\
\hline \multicolumn{3}{|l|}{ Área geográfica } & \multicolumn{3}{|c|}{ Índice de riqueza (quintiles) } \\
\hline Urbano & 280 & 45,0 & \multirow{4}{*}{$\begin{array}{l}\text { Primer (inferior) } \\
\text { Segundo } \\
\text { Tercer }\end{array}$} & \multirow{2}{*}{364} & \multirow{2}{*}{58,5} \\
\hline Rural & 342 & \multirow[t]{2}{*}{55,0} & & & \\
\hline & & & & 130 & 20,9 \\
\hline \multicolumn{3}{|l|}{ Etnia } & & 83 & 13,3 \\
\hline Indígena & 231 & 37,1 & \multirow{2}{*}{$\begin{array}{l}\text { Cuarto } \\
\text { Quinto (superior) }\end{array}$} & \multirow{2}{*}{$\begin{array}{l}32 \\
13\end{array}$} & \multirow{2}{*}{$\begin{array}{l}5,1 \\
2,1\end{array}$} \\
\hline No indígena (otra etnia) & 391 & 62,9 & & & \\
\hline
\end{tabular}




\section{Cuadro 1.}

Características sociodemográficas y económicas de la población en estudio

\begin{tabular}{|c|c|c|c|c|c|}
\hline Característica & $\mathbf{N}$ & $\%$ & Característica & $\mathrm{N}$ & $\%$ \\
\hline Parto prematuro & & & \multicolumn{3}{|c|}{ Personas en el hogar } \\
\hline No & 525 & 93,1 & $<6$ personas & 265 & 42,6 \\
\hline $\mathrm{Si}$ & 39 & 6,9 & 6 a 9 personas & 271 & 43,6 \\
\hline & & & 10 personas o más & 86 & 13,8 \\
\hline \multicolumn{3}{|l|}{$\begin{array}{l}\text { Inscrito en programa de } \\
\text { crecimiento y desarrollo }\end{array}$} & \multicolumn{3}{|c|}{ Servicio de alcantarillado } \\
\hline No & 222 & 39,4 & $\mathrm{Si}$ & 228 & 36,7 \\
\hline $\mathrm{Si}$ & 342 & 60,6 & No & 394 & 63,3 \\
\hline Ha lactado & & & \multicolumn{3}{|c|}{ Servicio de acueducto } \\
\hline No & 11 & 2,0 & $\mathrm{Si}$ & 338 & 54,3 \\
\hline $\mathrm{Si}$ & 553 & 98,1 & No & 284 & 45,7 \\
\hline Orden de nacimiento & & & \multicolumn{3}{|c|}{ Servicio de gas natural } \\
\hline Primer hijo & 157 & 27,8 & $\mathrm{Si}$ & 219 & 35,2 \\
\hline 2 a 3 & 223 & 39,5 & No & 403 & 64,8 \\
\hline 4 a 5 & 110 & 19,5 & & & \\
\hline \multirow[t]{3}{*}{6 y más } & 74 & 13,1 & \multicolumn{3}{|c|}{ Servicio de energía eléctrica } \\
\hline & & & $\mathrm{Si}$ & 449 & 72,2 \\
\hline & & & No & 173 & 27,8 \\
\hline
\end{tabular}

Fuente: Cálculo de los autores a partir de datos de la ENSIN 2010 
Figura 1. Prevalencia de desnutrición global por características sociodemográficas y económicas

$$
30
$$

28

26

24

22

20

18

วำ

16

14

12

10

8

6

4

2

0

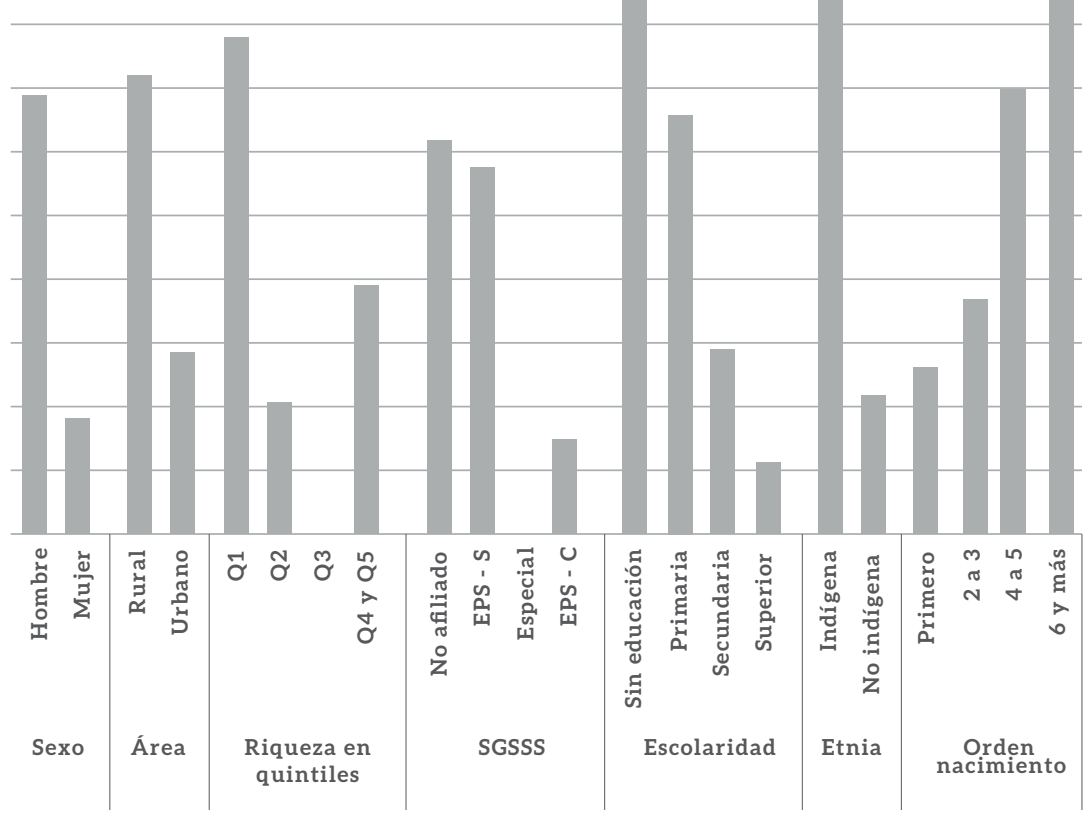

Desnutrición Global

Fuente: Cálculo de los autores a partir de datos de la ENSIN 2010 


\section{Cuadro 2.}

Modelo logit de determinantes de la desnutrición global en La Guajira.

\begin{tabular}{|c|c|c|c|}
\hline Variables explicativas & Odds Ratio & IC95\% & $p$ \\
\hline Hombre & 1,68 & $0,94-2,99$ & 0,08 \\
\hline Área urbana & 2,53 & $0,79-8,11$ & 0,12 \\
\hline Edad en meses & 0,73 & $0,38-1,40$ & 0,34 \\
\hline Etnia indígena & 1,68 & $0,67-4,19$ & 0,26 \\
\hline Parto prematuro & 1,32 & $0,33-5,33$ & 0,70 \\
\hline Programa de crecimiento y desarrollo & 1,48 & $0,82-2,65$ & 0,19 \\
\hline \multicolumn{4}{|l|}{$\begin{array}{l}\text { Orden de nacimiento (Referencia } \\
\text { primer hijo) }\end{array}$} \\
\hline 2 a 3 & 1,42 & $0,64-3,17$ & 0,38 \\
\hline 4 a 5 & 2,01 & $0,69-5,83$ & 0,20 \\
\hline 6 y más & 4,07 & $1,50-10,99$ & 0,01 \\
\hline \multicolumn{4}{|l|}{$\begin{array}{l}\text { Número de personas en el hogar } \\
\text { (Referencia }<6 \text { personas) }\end{array}$} \\
\hline Entre 6 y 9 & 1,20 & $0,53-2,72$ & 0,66 \\
\hline 10 personas o más & 0,99 & $0,36-2,68$ & 0,98 \\
\hline Pobreza & 0,79 & $0,18-3,50$ & 0,75 \\
\hline Energía eléctrica & 0,37 & $0,13-1,05$ & 0,06 \\
\hline Gas natural & 0,86 & $0,16-4,75$ & 0,86 \\
\hline Acueducto & 0,99 & $0,47-2,11$ & 0,99 \\
\hline Alcantarillado & 0,16 & $0,05-0,57$ & 0,01 \\
\hline \multicolumn{4}{|l|}{$\begin{array}{l}\text { Escolaridad de la madre (Referencia } \\
\text { sin educación) }\end{array}$} \\
\hline Primaria & 1,09 & $0,54-2,21$ & 0,80 \\
\hline Secundaria & 0,97 & $0,25-3,83$ & 0,97 \\
\hline Superior & 0,66 & $0,12-3,46$ & 0,62 \\
\hline \multicolumn{4}{|l|}{$\begin{array}{l}\text { Afiliación al SGSSS (Referencia } \\
\text { afiliado a EPS-C) }\end{array}$} \\
\hline EPS (Subsidiado) & 1,21 & $0,32-4,51$ & 0,77 \\
\hline Régimen especial & 1,00 & & \\
\hline No está afiliado & 1,12 & $0,26-4,74$ & 0,88 \\
\hline
\end{tabular}

Fuente: Cálculo de los autores a partir de datos de la ENSIN 2010 